\title{
STUDYING OPERATOR BEHAVIOUR DURING A SIMPLE BUT SAFETY CRITICAL TASK
}

\author{
Hans H. K. Andersen and Gunnar Hauland \\ Ris $\varnothing$ National Laboratory, Denmark and DNV, Norway
}

\begin{abstract}
A loss of sufficient Situation Awareness may lead to human errors, possibly resulting in accidents. Situation Awareness is often conceptually described in terms of operators' correct perception and understanding of a situation. It is though becoming increasingly clear, however, that team SA is an important concept as well. An initial idea for a continuous measure of Team Situation Awareness was tried out in a small technical pilot study conducted in the nuclear reactor control room at Risø. In our present study we seek to develop integrative methods combining eye-movement tracking data with other behavioral data. The study described in this paper is a pilot study, which seeks to establish the feasibility of applying these methods of measurement and analysis, not their validity.
\end{abstract}

Key words: Team Situation Awareness, Collaborative Work, Eye-Movement Tracking

\section{INTRODUCTION}

During millennia, evolution has granted humans an extremely effective perceptual-motor system serving control of the dynamic interaction with the environment and its inhabitants. In natural environments, all observable information is available all of the time. Thus, the actor can vary the level of abstraction at which the environment will be perceived at will. Listening to the sound of the wind in the trees helps us in understanding the weather even though we are focusing entirely on other activities. Likewise in the periphery of our attention, we are aware of activities of colleagues in our office, for example, by listening to footsteps outside our office door, listening in to 
small talk activities, discussions, etc. Also we signal our own availability in opening or closing our office doors.

Behavioral studies of decision-making during the natural flow of work have identified some basic characteristics of team situation awareness. Expert know-how and rules-of-thumb depend on adaptation to a work environment governed by an empirical correlation of convenient cues with successful acts. During normal familiar work, actors are immersed in the context for long periods; they know the flow of activity and the useful action alternatives by heart. They therefore do not base their actions on rational situation analysis, they do not have to base decisions on integration of a defining set of situation attributes (data) before acting in a familiar situation. Instead, they will seek no more information than is necessary for discrimination among the perceived alternatives for action in the particular situation. Consequently, they ask very biased questions to the environment and, consequently, when work situations change reliance on the usual convenient cues will lead to failure

The notion of team situation awareness in cooperative work settings has been emphasized by Rochlin et al. (1987) in their study of normal work practice onboard an aircraft carrier. Their findings point to the importance of the role of fringe-consciousness and an updated, tacit context awareness for a high cooperative reliability. Also within the CSCW community it has been frequently recognized that people's awareness of current work situations is an important characteristic of cooperative work. As such it has been shown that in many cooperative work settings it is possible to maintain situation awareness by the rich interaction, communication, and perceptive modalities of everyday social life. The study of Line Control Rooms on the London Underground (Heath and Luff, 1992) shows how actors maintain fluent reciprocal awareness regarding other actors' activities. In doing so the actors monitor each other's activities by overhearing other actors' radio or telephone conversations. Also they attract attention to activities, which are less visible to others, for example, when working with timetables and logs, by reading or thinking aloud or even by humming, singing, feigning momentary illness etc. In distributed cooperative work settings it is very difficult to maintain the same kind of situation awareness due to separation in perhaps both time and place.

\section{TEAM SITUATION AWARENESS AND TEAMWORK}

A loss of situation awareness may lead to human errors, possibly resulting in accidents. Situation awareness is often conceptually described in 
terms of operators' correct perception and understanding of a situation. This conception of individual situation awareness has been summarized by saying that an operator should be able to (1) recognize the relevant elements in a situation, (2) understand how these elements are interacting and, on the basis of this understanding, (3) predict the system status into the immediate future (Endsley, 1993; 1995). A diminished degree of awareness at any of the three levels will typically compromise performance.

It is typical of complex technical real time systems that they require, for the sake of safety and efficiency, more than one operator. When two or more operators are controlling a process, the collaborating collection of operators can be referred to as a team, i.e. the concept of team situation awareness should include inter-personal aspects of awareness. So, relative to each individual operator, his or her current model of the task domain will also include how other team members perceive and understand the situation and how they understand his current knowledge. Thus, in a sense team situation awareness is a component of individual situation awareness: operators must to some extent be aware of each other's tasks and of each other's awareness of those tasks. In short, therefore, team situation awareness involves team members' mutual knowledge about their task domain (See Andersen et al., 2001 for details on this point).

SA may be assessed by either subjective measures (involving operators' or expert observers' qualitative evaluation of performance and behaviors) or objective ones directed at subjects' responses and task directed behaviors. In this paper and in the study we refer to - we have concentrated efforts on objective measures- For several reasons we prefer to apply measures that do not involve an interruption of operators' task. That is, we have focused on assessment methods that involve measures that are made continuously across the evolvement of the task scenario. In a study of pilots visual behavior and its relations to team situation awareness we found that the pilots showed a high similarity in their scanning behavior across normal and abnormal situations and a high homogeneity within these situations.

Based on interviews with the operators we have sought to define such set of ideal visual behaviors that could allow us to compare this norm with the observed visual behavior. Thus, we hypothesize that the degree of correspondence (i.e., in terms of percentage) between a pre-defined ideal visual behavior and the observed visual behavior may be able to constitute a measure of situation awareness. As will be explained below, the team aspect will then be added to this measure by including behaviors, which involve the perception of, and interaction with fellow team members.

While it could be possible to define an ideal visual scanning behavior for the completion of a certain task, this need not be the case when it comes verbal and non-verbal coordination activities. As also mentioned above such 
activities may not in all cases follow ideal paths, since there might be a need deviate from, for example, procedures just to make things work in a given situation.

While operators do not always access system and task information visually, the situation awareness measure is based on operators' accessing visually presented information (Hauland, 2003). A range of measurable psycho-physiological patterns and variables (including those that originate from the use of eye-movement tracking) are underdetermined when it comes to interpreting what they might indicate about cognitive processes. That is to say, data thus obtained must be disambiguated, categorized and interpreted in the light of additional data about what cognitive processes and actions are likely in the domain under consideration.

The data analyses of such information gathering include units of analysis at this level of meaning, i.e., at the semantic level. At the same time there is a need for having data that potentially represent meaning in order to guide analyses of physiological data. Visual behavior does not by itself reveal or signify what the subject is thinking and intending rather, that semantic analysis is required to achieve this. Operators communicate through the process interface, i.e. they may infer what the other operators are aware of by watching key parameters in the process interface. Such Areas of Interest (AOI) may be substitutes for questions concerning each process parameter. We therefore pursue a method of defining AOI's relevant to team situation awareness and, subsequently, of measuring the line of gaze towards these AOI's by using eye-movement tracking. For the verbal behavior of operators we have selected an aspect that in previous studies have proven to correlate well with performance and task outcomes. Thus, communication may reveal how well the operators understand the developing situation. In studies conducted by Ris $\varnothing$ and the Danish Maritime Institute (Andersen et al., 1996), a significant correlation was found between crews' communication related to future system states and performance in simulated (ship maneuvering) tasks.

What the above means, in terms of trying to measure operators team situation awareness during system control activities is that it is necessary to have methods capable of combining the analysis of (i) visual and other behavioral data; (ii) subjective (where raw data observers' or subjects' own interpretations), and objective data (where raw data are recordings of directly observable behavior), and (iii) data representing both voluntary/intentional behavior (actions) and psycho-physiological data (automatic, micro-behavioral data that do not directly represent intentions).

In our present study we seek to develop such integrative methods combining eye-movement tracking data with elements of, first the Cognitive Systems Engineering framework developed at Ris $\varnothing$ National Laboratory (Rasmussen et al, 1994) for analyzing operators' cognitive activities and 
second the sociological frameworks for the analysis of everyday social nonverbal communication modalities (Andersen, 1997). While Cognitive Systems Engineering is relatively well known within the area of Human Error Safety and Systems Development the sociological approach to the study of team situation awareness activities is less recognized and may require a little attention here.

In engaging in teamwork actors generally become mutual dependent. They cannot fulfill the tasks on their own, so they have to rely on the contribution of other actors applying their different capacities, competencies, strategies and perspectives. Given their interdependence they need, in some way, to articulate their individual activities in joining their efforts. The term "articulate" in this context comes from the work of Strauss (1985), and Gerson and Star (1986). In this sense articulation means to allocate, coordinate, schedule, interrelate, integrate, etc., individual activities according to the dimensions of who, where, when, how, what, etc. The articulation work can be considered a type of second order activities or overhead cost in terms of the use of resources or time. The actors engage in these overhead activities because they would not on an individual basis be able to accomplish a certain task.

Teamwork is constituted by the fact that multiple actors are interdependent in their work. In other words, they are working in the same "field of work", that is, they are transforming and controlling a conglomerate of mutually interacting objects and processes. Thus, all teamwork involves and, indeed, is based upon interaction through changing the state of a common field of work. What one actor $\mathrm{A}$ is doing is of import to $\mathrm{B}$ and $\mathrm{C}$ in doing their work. The other actors $\mathrm{C}$ and $\mathrm{B}$ may to some extent be able to infer what $\mathrm{A}$ is doing from the changing state of the field of work. However, while collaborating via changing the state of the field of work is basic to all teamwork, it is rarely adequate. In fact, articulation of teamwork involves and, indeed, requires a vast variety of social modes of interaction that are combined and meshed dynamically and seamlessly in accordance with the specific requirements of the unfolding work situation and the means of communication available. As we see it there are four main interaction categories or modes of interaction:

Maintaining reciprocal awareness: The team could be involved in synchronous activities, by monitoring colleges' location in a room, and to monitor their activities. Moreover, they could be engaged in explicitly making their own activities publicly visible to teammates by thinking aloud, humming, etc.

Directing attention: Actors attract the attention of team-mates to focus on certain features or emerging problems in the field of work by, for 
example, to position certain items in certain ways, by pointing or nodding at particular items.

Assigning tasks: Actors could for example allocate a task by nodding at a work object or by stating a verbal request.

Handing over responsibility of processes in the field of work, for example, by passing on the work object in question, or the interface of a control mechanism.

These social modes of interaction are combined and meshed dynamically and fluently to meet the requirements of a specific situation. The different modes of interaction cannot be ordered in any simple kind of way but is possible to point at a limited number of prominent dimensions of the modes of interaction. Some examples:

Unobtrusive versus obtrusive, that is, some modes of interaction can be disruptive in nature in relation to a colleagues' line of work, while others are very conspicuous and therefore permit colleagues to carry on working.

Embedded versus symbolic, that is, to embed cues in highlighting certain items belonging to the field of work by for example marking them versus using a symbolic representation of the cues, which through its abstract function offers a higher degree of freedom regarding the manipulation of the cues.

Ephemeral versus persistent, that is, shared situational awareness only appears during the course of work and then disappears without leaving any trail to track. It is for example not immediately possible to trace activities like monitoring co-workers activities or to make one's own activities publicly visible.

The study described in this paper is a pilot study, which seeks to establish the feasibility of applying these methods of measurement and analysis, not their validity. The initial ideas for a continuous measure of team situation awareness were tried out in a small technical pilot study conducted in the nuclear reactor control room at Ris $\varnothing$ (a $12 \mathrm{MW}$ research reactor). The study was carried out during normal operation of the reactor control room focusing on operators' co-ordination of tasks in achieving the desired level of safety and efficiency for running the reactor.

\section{THE PILOT STUDY}

The data derived from the pilot study described here have been subjected to the integrative analysis described above. Since the data recordings associated with this type of eye-movement tracking are rather complex in themselves we need to describe them briefly. 
Ris $\varnothing$ has invested in new laboratory equipment enabling faster and more flexible analyses of many types of data, visual data in particular. The use of these data recording and analyses systems is highly skill based and the set-up of components in the field is not straightforward. It was therefore decided to explore the usability of major components of the new laboratory equipment in the field with the specific consideration in mind that these types of data may be used for the analysis of team situation awareness.

The two main objectives of this technical pilot study were to (1) gain hands-on experience with the eye tracking and the analysis systems under conditions where this equipment had to be operated in the field during real (non-interruptible) scenarios and (2) to acquire experience about the implementation of the suggested measures to be combined into an assessment of team situation awareness.

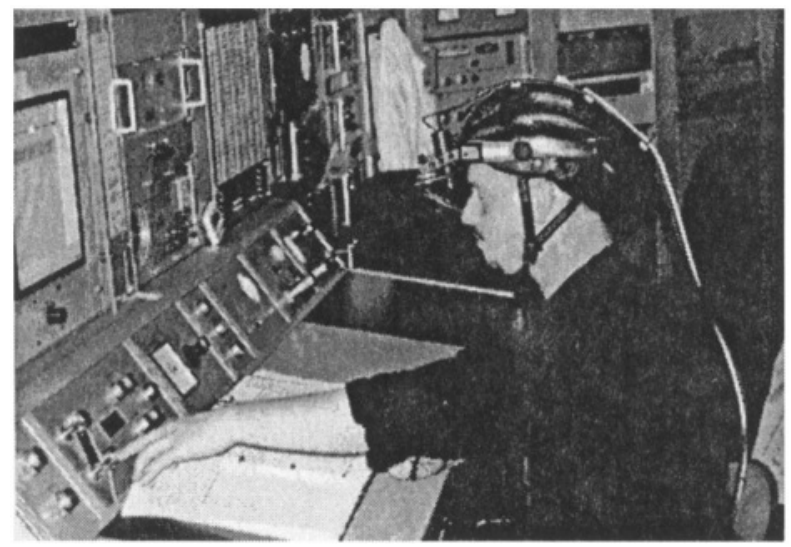

Figure 1. A nuclear control room operator wearing the eye-tracking helmet.

The selected target task in the nuclear research reactor (configuration of neutron flux by inserting and removing of neutron absorbing rods) is typically performed at intervals of 48 hours during normal operations. The exchange of rods aims at optimizing the configuration of the core. The task, which requires one operator at the top of the reactor to adjust the rods, and one operator in the control room to monitor instruments, lasts from 2-5 minutes (excluding preparations). The two teammates have to co-ordinate their tasks closely in order to adjust the reactor in a safe way. The removal of the rod has been done very smoothly and not too fast. Failing to do so will cause the reactor to shutdown automatically. For the pilot study data was collected through:

a) Combined head- and eye-movement tracking from the operator in the control room.

b) Video recordings of the operator at the top of the reactor.

c) Video recordings of the $3 \mathrm{D}$ model of the instrumentation in the control room (mixed with eye point gaze data). 
d) Audio recordings from both operators.

e) Questionnaire.

f) Debriefing with the group of operators.

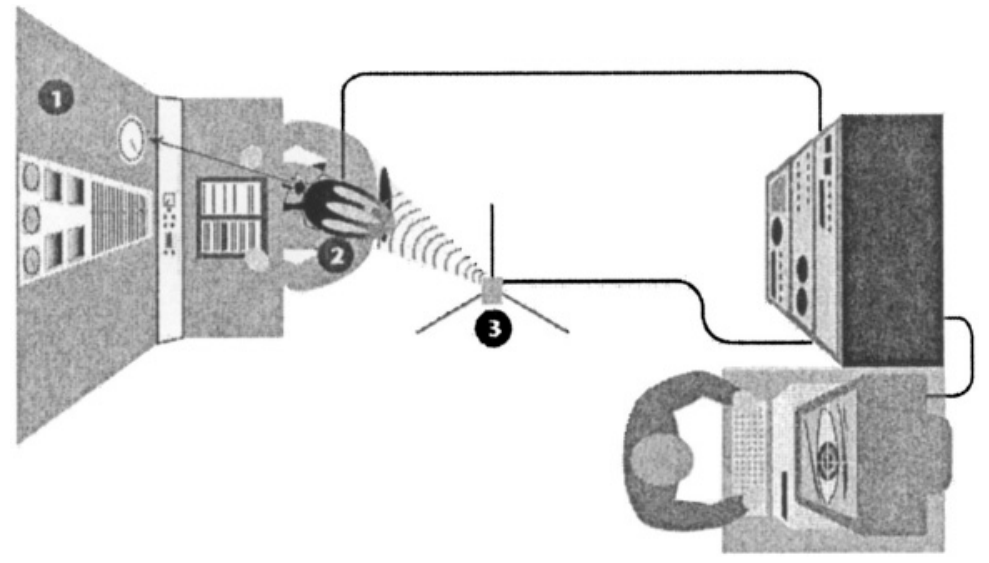

Figure 2. The technical set-up of equipment.

Figure 2 illustrates the overall set-up in the reactor control room with one of the operators and one of the researchers. The second operator is located on the top of the reactor, but is visible to his colleague through the monitor in the control room.

1. The reactor instruments, monitored only by the operator in the control room, display how the neutron flux in the reactor core is changing as a consequence of the team-mate's manual removal of neutron absorbing rods. The operators communicate through the intercom.

2. The operator wears the eye-tracking equipment. An effector placed on the top of the helmet gives the position of the head relative to the environment using a magnetic field. Together these enable continuous measurement of what instruments the operator is looking at. Both operators are wearing wireless microphones.

3. The magnetic tracking system combined with a laser pointer tool is used for building a 3D computer-model of the control room. The magnetic transmitter is the reference point for the effector mounted on the helmet, enabling integrated eye- and head tracking displayed in the model. The advantage is that eye-movement data can be analyzed automatically. 


\section{RESULTS}

Pre-study interviews indicated that the operators used most of their time during the task to monitor the Fine Control Rod meter (FCR), the effect meter, doubling time meter and the monitor (video of top of reactor). The fixation frequencies for the operators showed another picture. The operators looked outside these instruments more than 20 times on average during the operation. They mostly used the FCR for monitoring the task (15 fixations an average). They had 3 fixations on the effect meter, 6 fixations on the doubling time meter; and only one fixation on the monitor (video from top of reactor). The total duration of eye-point of gaze during the operation the operators looked outside the mentioned instruments for more than $40 \%$ of the time on an average, while gazing at the FCR in $42 \%$, the effect meter for $3 \%$, the double time meter $12,5 \%$, and the monitor $2,5 \%$ during the task (see Figure 3).

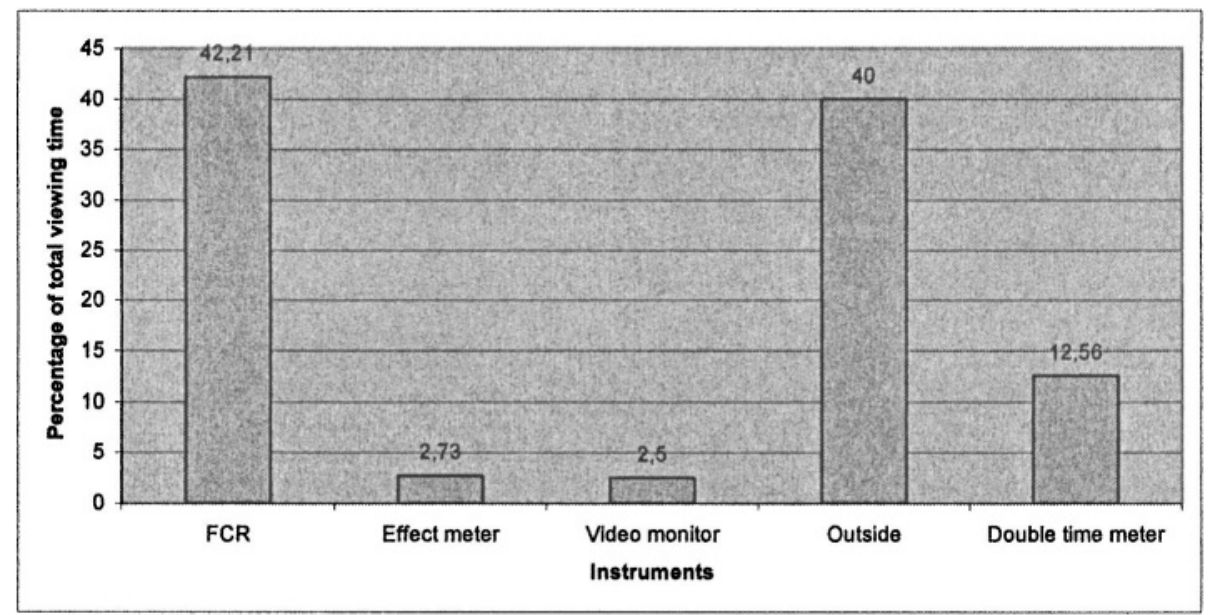

Figure 3. Viewing time on areas of interest.

This means that although the operators in the control room had the possibility to watch the actual removal of the rod they prefer not surprisingly to monitor the task through the different meters. The data shows that they mostly use the monitor to see that the operator is at the top of the reactor, so they can tell him (through an intercom) to start the task.

Figure 4 shows a model of the task. The two involved operators initiate the task in the control room in coordinating who is to do what. The operator on the top (OP) prepares for the removal of the rod, while the operator in the control room (OC) goes through the logbook to check the size of the rod, and date and time for removing the rod. While doing this he looks at the monitor a couple of times to see how OP progresses. When OP has finished 
the preparation for removal he positions his body in a certain way to in a non-verbal way to communicate through the video camera to OC that he is ready to pull the rod out. The reason for this is that, he is not able to use the intercom from where he is standing (to speak through the intercom the OP needs to press a button because it is a simplex based devise). The OC sees that OP is ready to pull and issues a "start" command. While OP pulls the rod, OC monitors the instruments. When OP is finished he walks to the intercom and issues a "finish" command. Then OC update the logbook, while OC cleans up after the removal of the rod. The team situation awareness aspects of this task is most clear, we think, in that the OP knows that he is being monitored via the video channel. The OP also knows that the OC knows that when he (the OP) positions his body to communicate readiness for pulling the rod, so this is a signal to issue the start command. In terms of the social modes of interaction (discussed in Section 2) conveying the team situation awareness of the two operators, the operators seek to maintain reciprocal awareness. That is, in their synchronous activities, the operator in the control room monitors his college's location on the top of the reactor, while the operator on the top explicitly makes his own activities publicly visible to his teammate and communicates readiness to carry on his task by placing his body in certain way. If we look at the dimensions of the social modes of interaction the operators' team situation is maintained in unobtrusive way and therefore permit the teammates to carry on working that in the given situation can be considered as optimal strategy. The coordination activities are also ephemeral in the sense that team situational awareness only appears during the course of work, which could be considered as a less optimal strategy, for example, if less experienced operators where to coordinate the task it could be considered to use a checklist to control the coordination activities.

This is of course a very simple task and there might be other ways of interpreting the task. Also we are of course aware that the technology available in the 30-year-old reactor, in certain ways, provokes certain awareness activities. This could also be the case in more advanced systems, but then on a very different level.

In the questionnaires, operators were asked to describe the success of the rod removal. They all agreed that the task could not have been performed better. All tasks were performed according to regulations and there were no shutdowns.

Operators had not worked together on this particular task before (due to summer vacation), but they had all worked together for a long time on other tasks at the reactor. This may have played a role in the communication, since no misunderstandings were produced. 
Based on the visual and verbal behavior/questionnaires/recorded instruments, operators had no problems performing the task. The removal of neutron absorbing rods is a relatively simple task with respect to perception and understanding, but it is a safety critical task that has to be carried out manually. The fact that the task has to fine-tuned with activities in the control room demands the operators to maintain a sufficient level of team situation awareness in order not do any mistakes.

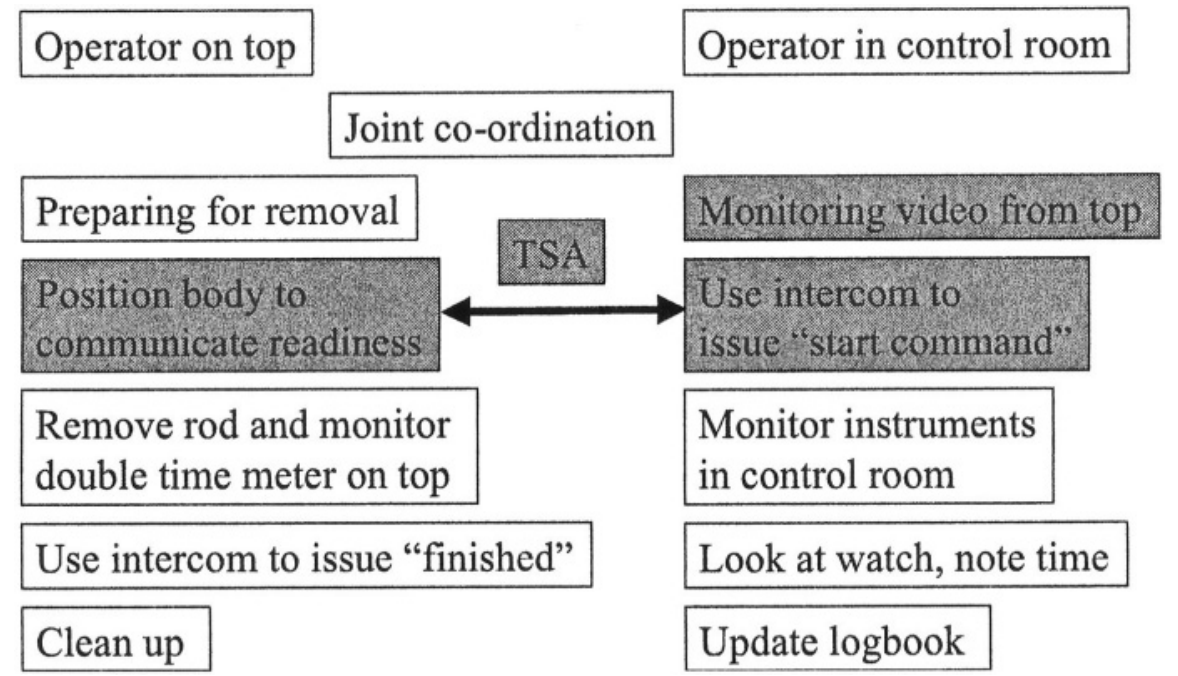

Figure 4. A model of team situation awareness during pulling of the control rod. Gray-scaled areas indicate team situation awareness activities.

As a point of departure for de-briefing session we showed a video where we had included clip that seemed a bit more difficult than the rest. The debriefing-video consisted of a mix between our eye-movement tracking scene video recordings, the recordings of the $3 \mathrm{D}$ model and various location recordings. We focused on discussing situation awareness, but also discussed other issues. The main result from the de-briefing is summarized below:

There were sources of information not detectable through analyses of visual information gathering and verbal communication, like e.g. listening to the elevator driving the rods.

Operators did not look at the instruments they claimed to be looking at (both before the study and when watching the video).

All operators agreed on what information was important to solve the task.

However, the discussion revealed that operators, even the most experienced ones, disagreed with respect to the priority of information acquisition, i.e. exactly how to access the relevant information (from what instrument, for how long etc.). 


\section{CONCLUSION}

The de-briefing session showed us that it seems to be difficult to establish a standard means by which the operators acquire information during the rod removal task and no written procedure exists i.e. defining the norm for team situation awareness may be difficult. Although individual differences in the approach to information acquisition can be observed, these differences are not necessarily more or less correct. One feasible way to measure team situation awareness may be to look for the lowest common denominator for the information items, that the operators need to solve a task, and to avoid defining details concerning qualities of the operators information gathering activities like sequence and duration of the activities, except when there can be established a clear operational definition of these activities, e.g. where the operators activities under certain circumstances are specified in checklists. (See also Hauland 1996 on this issue).

Although operators did use instruments differently, they all relied on the same information. It was not possible from these example trials to observe variation neither in task performance nor intermediate activities like visual information gathering (type of information) and verbal communication. It seems like a (complex) measure of team situation awareness like the one proposed, is less useful for very simple tasks - tasks not likely to produce much variation.

Situation awareness is thought to be more than exceptional attention. It includes the integration of many elements in the situation, including the projection of how the situation will develop. One could ask if judging ordinal single variables like on/off, under/above calls for the type of overview we want to measure with team situation awareness. If it does, one could propose another explanation for the lack of variation: It is assumed that mental workload is tightly coupled with the concept of team situation awareness.

The relationship between workload and situation awareness is often claimed to constitute an inverse u-shaped curve: A low level of mental workload is associated with a low level of situation awareness, a medium level of mental workload is associated with a high level of situation awareness, and a very high level of mental workload is associated with a breakdown of situation awareness. Thus, if a very low level of task complexity is perceived, one would expect this to be reflected in a low level of mental workload, and consequently low situation awareness.

This explanation is probably not relevant here however, since all tasks were performed in accordance with regulations. It is more relevant, we think, to ask if this task really required team situation awareness in the way we have defined it. The difficulty in the current task - if any - is sensory- 
motoric (pull slowly) and co-ordination (stop pulling on command from the control room operator). It may be relevant also to ask about situation awareness in very simple task, but the complexity of the measure (resolution and number of units of analyses) must be in proportion to the tasks to be performed. Thus, the team situation awareness measure we wish to develop aims at measuring awareness of complex tasks, where complexity may be reflected in, e.g., number of situation elements and the type of relationship between these elements.

\section{REFERENCES}

Andersen, H. H K. (1997). Cooperative Documentation Production in Engineering Design: The 'Mechanisms of Interaction' Perspective, Topics in Cognitive Science and HCI, Centre for Cognitive Informatics. Roskilde University, (9).

Andersen, H.B.; Pedersen, C.R.; Andersen,H.H.K. (2001) Using eye tracking data to indicate team situation awareness. In: Usability evaluation and interface design: Cognitive engineering, intelligent agents and virtual reality. Proc. of HCI International 2001. Vol. 1. 9. Int. conf. on human-computer interaction. Smith, M.J.; Salvendy, G.; Harris, D.; Koubek, R.J. (eds.), (LEA, Inc., Mahwah, NJ, 2001) p. 1318-1322.

Andersen, H.B., Sørensen, P.K., Weber, S., and Sørensen, C. (1996): A study of the performance of captains and crews in a full mission simulator. Ris $\varnothing$ National Laboratory, Roskilde. Ris $\varnothing-$ R-916.

Endsley, M.R. (1993). Situation Awareness in Dynamic Human Decision Making. Proceedings of the 1st International Conference on Situational Awareness in Complex Systems, Orlando, February 1993.

Endsley, M.R. (1995) Towards a Theory of Situation Awareness. Human Factors, 37 (1), 3264.

Gerson, Elihu M., and Star, S.L. (1986), “Analyzing Due Process in the Workplace,” TOIS, vol. 4, no. 3, pp. 257-270.

Hauland, G. (2003) Measuring Team Situation Awareness by means of Eye Movement Data. In: Proceedings. Vol. 3. Human-centred computing. Cognitive, social and ergonomic aspects. 10. International conference on human-computer interaction (HCI International 2003), Crete (GR), 22-27 Jun 2003. Jacko, J.; Stephanidis, C. (eds.), (Lawrence Erlbaum Associates, Inc., Mahwah, NJ, 2003) p. 230-234.

Heath, C., and Luff, P. (1991), Collaborative Activity and Technological Design: Task Coordination in London Underground Control Rooms, in ECSCW '91. Proceedings of the Second European Conference on Computer-Supported Cooperative Work, ed. by L. Bannon, M. Robinson and K. Schmidt, Kluwer Academic Publishers, Amsterdam, 1991, pp. 65-80.

Rasmussen, J., Pejtersen, A.M., \& L.P., Goodstein. (1994). Cognitive Systems Engineering. New York: John Wiley.

Rochlin, G. I., La Porte, T. R., and Roberts, K. H. (1987) The Self designing High-Reliability Organization: Aircraft Carrier Flight Operations at Sea, Naval War College review, Autom.

Strauss, A., Fagerhaugh, S., Suczek, B. and Wiener, C. (1985) Social Organization of Medical Work, University of Chicago Press, Chicago and London. 